\title{
Collocation with Integer Trend
}

\section{P.J.G. Teunissen}

Delft Institute of Earth Observation and Space systems (DEOS), Delft University of Technology, Kluyverweg 1, 2629 HS Delft, The Netherlands, e-mail: P.J.G.Teunissen@TUDelft.nl

\begin{abstract}
Collocation is a popular method in geodesy for combining heterogeneous data of different kind. It comprises adjustment, interpolation and extrapolation as special cases. Current methods of collocation apply however only if the trend parameters are real valued. In the present contribution we will generalize the theory of collocation by permitting the trend parameters to be integer valued. It will be shown how the collocation formulae change when the integerness of the trend parameters is taken into account. We will also address the problem of evaluating the quality of the collocation results. The quality of the collocation results is usually described by the so-called error covariances. We will show how the error covariances change due to the integerness of the trend. But we also show that the approach based on error covariances does not give an adequate quality description of the collocation results in case of an integer trend. How this approach needs to be generalized is also presented.
\end{abstract}

Keywords. Collocation, trend-signal-noise model, integer least-squares

\section{Introduction}

Least-squares collocation is a popular method in geodesy for combining heterogeneous data of different kind (Krarup, 1969, Moritz, 1973, Dermanis, 1980, Sanso, 1986). It comprises adjustment, interpolation and extrapolation as special cases. The underlying model of least-squares collocation consists in its general form of three terms: a trend, a signal and a noise term. The trend is then often further parametrized in a set of unknown parameters. This so-called trend-signal-noise model is quite general and it encompasses many of the conceivable geodetic measurements (Moritz, 1980). The current collocation methods are however only applicable if the trend parameters are real valued. In the present contribution we will generalize the theory of collocation by permitting some or all of the trend parameters to be integer valued.

We first give a brief review of the method of collocation when the trend parameters are real-valued. This includes the part where the observable vector is separated in the trend, the signal and the noise, as well as the part in which an unobservable vector, such as the signal for instance, is predicted. We then show how the collocation formulae change when the integerness of the trend parameters is taken into account. It is shown that the general structure of collocation remains unaffected, but that an additional computational step based on the principle of integer leastsquares needs to be inserted. We also address the problem of evaluating the quality of the collocation results. In the classical case the quality of the collocation results is described by the so-called error covariances. We show how the error covariances change due to the integerness of the trend. But we also show that the approach based on error covariances does not give an adequate quality description of the collocation results in case of an integer trend. Instead one will have to make use of the joint probability density function of the collocation error. The error distribution will not be normal even if the input data are normally distributed. The distribution of the collocation error will also be given.

\section{Collocation}

\subsection{Trend, Signal and Noise Model}

\section{Separation of Trend, Signal and Noise}

In the trend-signal-noise model the observable vector $y$ is written as a sum of three terms, $y=t+$ $s+n$, with $t$ a deterministic, but unknown trend, $s$ a zero-mean random signal vector, and $n$ a zeromean random noise vector. The trend is usually further parametrized in terms of an unknown $p \times 1$ parameter vector $x$ as $t=A x$. The signal and noise vector are assumed to be uncorrelated and their variance matrices are given as $Q_{s s}$ and $Q_{n n}$, respectively. 
Thus we have

$$
y=A x+s+n
$$

with $Q_{y y}=Q_{s s}+Q_{n n}$. We assume the variance matrices to be positive definite and matrix $A$ to be of full column rank. Application of the least-squares collocation minimization principle (Moritz, 1973), gives

$$
\begin{gathered}
\hat{x}=\left(A^{T}\left(Q_{s s}+Q_{n n}\right)^{-1} A\right)^{-1} . \\
A^{T}\left(Q_{s s}+Q_{n n}\right)^{-1} y \\
\hat{s}=Q_{s s}\left(Q_{s s}+Q_{n n}\right)^{-1}(y-A \hat{x}) \\
\hat{n}=Q_{n n}\left(Q_{s s}+Q_{n n}\right)^{-1}(y-A \hat{x})
\end{gathered}
$$

Note that the separation of trend, signal and noise is reflected in the identity $y=A \hat{x}+\hat{s}+\hat{n}$. Also note that $\hat{e}=\hat{s}+\hat{n}=y-A \hat{x}$ is the least-squares residual vector.

\section{Predicting an Unobservable Vector}

Often one can extend the trend-signal-noise model so as to hold true for an unobservable vector $y_{0}$ as well. This gives

$$
y_{0}=A_{0} x+s_{0}+n_{0}
$$

in which $A_{0}$ is a given $m_{0} \times p$ matrix and $s_{0}$ and $n_{0}$ are uncorrelated zero-mean random vectors, with variance matrices $Q_{s_{0} s_{0}}$ and $Q_{n_{0} n_{0}}$, respectively. The two signal vectors, $s_{0}$ and $s$, are assumed correlated $\left(Q_{s_{0} s} \neq 0\right)$, whereas the two noise vectors, $n_{0}$ and $n$, are (usually) assumed to be uncorrelated $\left(Q_{n_{0} n}=0\right)$. Application of the least-squares collocation principle gives

$$
\begin{aligned}
& \hat{y}_{0}=A_{0} \hat{x}+Q_{s_{0} s}\left(Q_{s s}+Q_{n n}\right)^{-1}(y-A \hat{x}) \\
& \hat{s}_{0}=Q_{s_{0} s}\left(Q_{s s}+Q_{n n}\right)^{-1}(y-A \hat{x}) \\
& \hat{n}_{0}=0
\end{aligned}
$$

Note that $A_{0} \hat{x}$ is the least-squares estimator of the mean of $y_{0}$. Thus if $s_{0}$ and $s$ are uncorrelated, then the predictor of $y_{0}$ coincides with the estimator of its mean. Also note that the predictor of the trend plus signal, $A_{0} x+s_{0}$, is identical to the predictor of $y_{0}$. Both are given as $A_{0} \hat{x}+\hat{s}_{0}$. In general this is not the case. In the present situation, the two predictors coincide since the noise vector $n_{0}$ was assumed to be uncorrelated with $s$ and $n$. For the same reason the predictor of $n_{0}$ is identically zero.

\subsection{Error Variance Matrices}

In order to judge the prediction quality of collocation, we need to consider the prediction errors. The prediction error of $\hat{y}_{0}$ is defined as $\hat{\epsilon}_{0}=y_{0}-\hat{y}_{0}$. It is a zeromean random vector, $E\left(\hat{\epsilon}_{0}\right)=0$. Thus the predictor $\hat{y}_{0}$ is unbiased. The variance matrix of $\hat{\epsilon}_{0}$ is called the error variance matrix of $\hat{y}_{0}$. It will be denoted as $P_{\hat{y}_{0} \hat{y}_{0}}$ and it should not be confused with the variance matrix of $\hat{y}_{0}$. To determine $P_{\hat{y}_{0}} \hat{y}_{0}$, we first write $\hat{\epsilon}_{0}=y_{0}-\hat{y}_{0}$ as $\hat{\epsilon}_{0}=\left(y_{0}-Q_{y_{0}} Q_{y y}^{-1} y\right)-\left(A_{0}-\right.$ $\left.Q_{y_{0} y} Q_{y y}^{-1} A\right) \hat{x}$. Note that the first bracketed term is uncorrelated with $y$. Since $\hat{x}$ is a linear function of $y$, it follows that the first bracketed term is also uncorrelated with $\hat{x}$. Application of the error propagation law gives therefore

$$
\begin{aligned}
& P_{\hat{y}_{0} \hat{y}_{0}}=Q_{y_{0} y_{0}}-Q_{y_{0} y} Q_{y y}^{-1} Q_{y y_{0}}+ \\
& \quad\left(A_{0}-Q_{y_{0} y} Q_{y y}^{-1} A\right) Q_{\hat{x} \hat{x}}\left(A_{0}-Q_{y_{0} y} Q_{y y}^{-1} A\right)^{T}(5)
\end{aligned}
$$

in which $Q_{\hat{x} \hat{x}}$ is the variance matrix of $\hat{x}$. The three terms on the right hand side of this expression can be understood as follows. Would $x$ be known and $y$ be absent, the error variance matrix would be given as $P_{\hat{y}_{0} \hat{y}_{0}}=Q_{y_{0} y_{0}}$. In this case the uncertainty is completely due to the uncertainty of $y_{0}$. But with the observable vector $y$ present and $x$ still known, the error variance matrix gets reduced to $P_{\hat{y}_{0} \hat{y}_{0}}=$ $Q_{y_{0} y_{0}}-Q_{y_{0} y} Q_{y y}^{-1} Q_{y y_{0}}$. The uncertainty reduces due to the contribution of $y$. But since $x$ is unknown, and has to be estimated, the error variance matrix gets enlarged by the third term.

With $Q_{y_{0} y_{0} \mid y}=Q_{y_{0} y_{0}}-Q_{y_{0} y} Q_{y y}^{-1} Q_{y y_{0}}$ and $A_{0 \mid y}=A_{0}-Q_{y_{0} y} Q_{y y}^{-1} A$, we can write the error variance matrix in compact form as

$$
P_{\hat{y}_{0} \hat{y}_{0}}=Q_{y_{0} y_{0} \mid y}+A_{0 \mid y} Q_{\hat{x} \hat{x}} A_{0 \mid y}^{T}
$$

When $A_{0}=0$, we can obtain an alternative expression for the error variance matrix and one which is expressed in the variance matrix of the least-squares residual vector. It is given as

$$
P_{\hat{y}_{0} \hat{y}_{0}}=Q_{y_{0} y_{0}}-Q_{y_{0} y} Q_{y y}^{-1} Q_{\hat{e} \hat{e}} Q_{y y}^{-1} Q_{y y_{0}}
$$

where $Q_{\hat{e} \hat{e}}=Q_{y y}-A Q_{\hat{x} \hat{x}} A^{T}$. This result applies, for instance, when $s_{0}$ plays the role of $y_{0}$.

A complete probabilistic description of the prediction error can be given once its probability distribution is known. If we assume that $y$ and $y_{0}$ are normally distributed, then - since all relations are linear - also the prediction error is normally distributed. Its distribution is then given as $\hat{\epsilon}_{0} \sim N\left(0, P_{\hat{y}_{0}} \hat{y}_{0}\right)$. 


\section{Collocation with Integer Trend}

\subsection{Trend, Signal and Noise Model}

We now extend the model of the previous section so as to include the option that the trend parameter vector $x$ is integer valued, $x \in Z^{p}$. We will assume that all trend parameters are integer valued. The results of this and the following sections can be generalized however to the case that some but not all of the trend parameters are integer valued.

For the separation of trend, signal and noise, application of the least-squares collocation principle, but now with the integer constraints included, gives

$$
\begin{aligned}
& \check{x}=\arg \min _{z \in Z^{n}}\|\hat{x}-z\|_{Q_{\hat{x} \hat{x}}}^{2} \\
& \check{s}=Q_{s s}\left(Q_{s s}+Q_{n n}\right)^{-1}(y-A \check{x}) \\
& \check{n}=Q_{n n}\left(Q_{s s}+Q_{n n}\right)^{-1}(y-A \check{x})
\end{aligned}
$$

with $\|\cdot\|_{M}^{2}=(\cdot)^{T} M^{-1}(\cdot)$. Compare with (2) and note that now $y=A \check{x}+\check{s}+\check{n}$. For the prediction of $y_{0}, s_{0}$ and $n_{0}$, we get

$$
\begin{aligned}
& \check{y}_{0}=A_{0} \check{x}+Q_{s_{0}}\left(Q_{s s}+Q_{n n}\right)^{-1}(y-A \check{x}) \\
& \check{s}_{0}=Q_{s_{0} s}\left(Q_{s s}+Q_{n n}\right)^{-1}(y-A \check{x}) \\
& \check{n}_{0}=0
\end{aligned}
$$

Compare with (4). Note that the structure of the collocation results remains the same. That is, the above results can be obtained form those of (2) and (4) by replacing $\hat{x}$ by $\check{x}$. For information on how the integer least-squares solution $\breve{x}$ can be computed, we refer to (Teunissen, 1993, 1995, de Jonge and Tiberius, 1996).

To see the above equations at work, consider the following two examples.

Example 1. Consider the single equation $y=a x+$ $s+n$, with scalar $a$ given, $x$ an unknown integer, and $s$ and $n$ the zero mean signal and noise, respectively. The integer least-squares estimator of $x$ is then given as $\check{x}=[y / a]$, in which ' $[\cdot]$ ' denotes rounding to the nearest integer. For the signal and the noise, we obtain $\check{s}=\frac{\sigma_{s}^{2}}{\sigma_{s}^{2}+\sigma_{n}^{2}}(y-a \check{x})$ and $\check{n}=\frac{\sigma_{n}^{2}}{\sigma_{s}^{2}+\sigma_{n}^{2}}(y-a \check{x})$, respectively. Thus fractions of the residual $y-a \check{x}$ are assigned to $\breve{s}$ and $\check{n}$. They get an equal share of the residual vector if the two variances are equal. $\bigcirc$

Example 2. As a trend-signal-noise model, we consider the single-frequency, single epoch, geometryfree GPS equations, based on double-differenced (DD) carrier phase and pseudorange. The carrier phase and pseudorange equations are given as $y_{1}=$ $\lambda x+\rho+s+n_{1}$ and $y_{2}=\rho-s+n_{2}$, with $x$ the unknown integer DD carrier phase ambiguity, $\lambda$ the known wavelength of the carrier phase, $\rho$ the unknown DD range, $s$ the residual ionospheric signal, and $n_{1}$ and $n_{2}$ the noise of the carrier phase and the pseudorange, respectively. If we eliminate the range $\rho$ by taking the difference of the two equations, we obtain after dividing by two, the single trendsignal-noise equation

$$
y=(\lambda / 2) x+s+n
$$

with $y=\left(y_{1}-y_{2}\right) / 2$ and $n=\left(n_{1}-n_{2}\right) / 2$. The integer least-squares estimator of $x$ is then given as $\check{x}=\left[\left(y_{1}-y_{2}\right) / \lambda\right]$. Now let $s_{0}$ be the ionospheric signal at another time instant. Then its predictor $\check{s}_{0}=$ $Q_{s_{0} s}\left(Q_{s s}+Q_{n n}\right)^{-1}(y-A \check{x})$ works out as

$$
\check{s}_{0}=\frac{2 \sigma_{s_{0} s}}{4 \sigma_{s}^{2}+\sigma_{1}^{2}+\sigma_{2}^{2}}\left(y_{1}-y_{2}-\lambda \check{x}\right)
$$

where $\sigma_{s}^{2}$ denotes the variance of the ionospheric signal, $\sigma_{s_{0} s}$ is the covariance between $s_{0}$ and $s$, and $\sigma_{1}^{2}$ and $\sigma_{2}^{2}$ are the variances of the DD carrier phase and pseudorange, respectively.

\subsection{Error Variance Matrices}

The prediction error of $\check{y}_{0}$ is defined as $\check{\epsilon}_{0}=y_{0}-\check{y}_{0}$. Note that it is a zero-mean random vector, provided that $E(\check{x})=x$ holds true. Thus if the integer leastsquares estimator $\check{x}$ is an unbiased estimator of $x$, then $E\left(\check{\epsilon}_{0}\right)=0$. It can be shown that $E(\check{x})=x$ holds true when $y$ is normally distributed (Teunissen, 1999).

The variance matrix of $\check{\epsilon}_{0}$ is the error variance matrix of $\check{y}_{0}$. It will be denoted as $P_{\check{y}_{0} \check{y}_{0}}$. To determine the error variance matrix, we write the prediction error similarly as before as $\check{\epsilon}_{0}=\left(y_{0}-\right.$ $\left.Q_{y_{0} y} Q_{y y}^{-1} y\right)-\left(A_{0}-Q_{y_{0} y} Q_{y y}^{-1} A\right) \check{x}$. But now it is not generally true anymore that the two terms on the right hand side of this expression are uncorrelated. This is due to the fact that $\breve{x}$ is a nonlinear function of $y$. Hence, we need to make some additional assumptions on the distributional properties of $y$ and $y_{0}$. In order to obtain a result which in structure is comparable to our previous result (6), we will assume that $y$ and $y_{0}$ are normally distributed. Then the first bracketed term is independent of $y$. And since $\check{x}$ is a function of $y$, it follows that the first bracketed term is also independent of $\check{x}$. Application of the error 
propagation law gives therefore

$$
P_{\check{y}_{0} \check{y}_{0}}=Q_{y_{0} y_{0} \mid y}+A_{0 \mid y} Q_{\check{x} \check{x}} A_{0 \mid y}^{T}
$$

with $Q_{\breve{x} \check{x}}$ the variance matrix of $\check{x}$. Compare with (6) and note that $Q_{\hat{x} \hat{x}}$ has been replaced by $Q_{\check{x} \check{x}}$.

When $A_{0}=0$, we can obtain an expression which in structure is similar to (7). It is given as

$$
P_{\check{y}_{0} \check{y}_{0}}=Q_{y_{0} y_{0}}-Q_{y_{0} y} Q_{y y}^{-1}\left(Q_{y y}-A Q_{\check{x} \check{x}} A^{T}\right) Q_{y y}^{-1} Q_{y y_{0}}
$$

But note that the term within brackets is now not the variance matrix of the integer least-squares residual vector, $Q_{\check{e} \check{e}} \neq Q_{y y}-A Q_{\check{x} \check{x}} A^{T}$.

Example 3. We can use (12) to obtain the error variance of the ionospheric predictor of Example 2. It is given as

$$
\begin{aligned}
P_{\check{s}_{0} \check{s}_{0}}= & \sigma_{s_{0}}^{2}-\left(\frac{2 \sigma_{s_{0} s}}{4 \sigma_{s}^{2}+\sigma_{1}^{2}+\sigma_{2}^{2}}\right)^{2} \\
& \times\left(4 \sigma_{s}^{2}+\sigma_{1}^{2}+\sigma_{2}^{2}-\lambda^{2} \sigma_{\check{x}}^{2}\right)
\end{aligned}
$$

in which $\sigma_{\breve{x}}^{2}$ is the variance of the integer estimator.

Although it is nice to know the first two moments of the prediction error $\check{\epsilon}_{0}$, this information is not sufficient to capture all the probabilistic properties of the prediction error. This is due to the fact that $\check{\epsilon}$ is not normally distributed, even if $y$ and $y_{0}$ are. Hence, with only the error variance matrix available one can only make approximate statements. One may use the multivariate version of the Chebyshev inequality, for instance, to obtain an upper bound on the tail probability of the distribution of $\check{\epsilon}_{0}$. Then for every $t>0$, the following bound holds,

$$
P\left(\left\|\check{\varepsilon}_{0}\right\|^{2} \geq t^{2}\right) \leq \operatorname{trace}\left(P_{\check{y}_{0} \check{y}_{0}}\right) / t^{2}
$$

where $\|\cdot\|$ denotes the standard norm.

\subsection{Distributional Results}

A complete probabilistic description of the prediction error can be given once its probability distribution is known. In this section we will present the distribution of $\check{\epsilon}_{0}$. Although a rigorous proof is outside the scope of the present contribution, we will make the result plausible. As before, we will assume $y$ and $y_{0}$ to be normally distributed.
The prediction error can be expressed as

$$
\check{\epsilon}_{0}=y_{0}-Q_{y_{0} \dot{y}} Q_{y y}^{-1} y-A_{0 \mid y} \check{x}
$$

It depends on the three random vectors $y_{0}, y$ and $\check{x}$. Each one of them contributes to the random behaviour of $\check{\epsilon}_{0}$. Would $\check{x}$ be nonrandom and equal to, say, $z$, then $\check{\epsilon}_{0}$ would be normally distributed with mean $A_{0 \mid y}(x-z)$ and variance matrix $Q_{y_{0} y_{0} \mid y}$. Hence, its probability density function (PDF) would then be given as

$$
\begin{aligned}
f_{\check{\epsilon}_{0} \mid \check{x}=z}(v) & =\frac{1}{(2 \pi)^{m_{0} / 2} \sqrt{\operatorname{det} Q_{y_{0} y_{0} \mid y}}} \times \\
& \exp \left\{-\frac{1}{2}\left\|v-A_{0 \mid y}(x-z)\right\|_{Q_{y_{0} y_{0} \mid y}}^{2}\right\}
\end{aligned}
$$

However, since $\check{x}$ is not deterministic, but a random vector, one needs to take its distributional properties into account as well. And since $\breve{x}$ is a nonlinear function of $y$, one can not expect the prediction error to be normally distributed.

Since $\breve{x}$ has integer outcomes only, its distribution will be a probability mass function (PMF). Let $f_{\hat{x}}(u)$ be the normal PDF of $\hat{x}$. The PMF of $\check{x}$ can then be shown to be given as

$$
P[\check{x}=z]=\int_{S_{z}} f_{\hat{x}}(u) d u, \quad \forall_{z} \in Z^{p}
$$

where $S_{z}$ is the pull-in region of the integer leastsquares estimator, see (Teunissen, 1999). With these probability masses and the PDF of (14), the PDF of the prediction error follows as the infinite sum $f_{\check{\epsilon}_{0}}(v)=\sum_{z \in Z^{p}} f_{\check{\epsilon}_{0} \mid \check{x}=z}(v) P[\check{x}=z]$. We therefore have

$$
\begin{aligned}
f_{\check{\epsilon}_{0}}(v) & =\sum_{z \in Z^{p}} \frac{P[\check{x}=z]}{(2 \pi)^{m_{0} / 2} \sqrt{\operatorname{det} Q_{y_{0} y_{0} \mid y}}} \times \\
& \exp \left\{-\frac{1}{2}\left\|v-A_{0 \mid y}(x-z)\right\|_{Q_{y_{0} y_{0} \mid y}}^{2}\right\}
\end{aligned}
$$

This result shows that the PDF of the prediction error is a multimodal distribution. It is an infinite sum of weighted and shifted versions of the PDF of (14). The weights are given by the probability masses of the PMF of $\check{x}$. Note that the PDF of the prediction error is symmetric with respect to the origin. This confirms that $E\left(\breve{\epsilon}_{0}\right)=0$. Also note, if the probability of correct integer estimation $P[\check{x}=x]$ approaches one, that the PDF of $\check{\epsilon}_{0}$ approaches the normal distribution with zero mean and variance matrix $Q_{y_{0} y_{0} \mid y}$. 
With the PDF of $\check{\epsilon}_{0}$ one can now describe the predictive quality of integer trend collocation. For instance, if one wants to obtain confidence regions for the prediction of the signal $s_{0}$, one uses (16) with $A_{0}=0$ and $Q_{y_{0} y_{0} \mid y}=Q_{s_{0} s_{0}}-Q_{s_{0} s}\left(Q_{s s}+\right.$ $\left.Q_{n n}\right)^{-1} Q_{s s_{0}}$. The confidence region follows then as the set $\left\{v \in R^{m_{0}} \mid f_{\check{\epsilon}_{0}}(v) \geq c\right\}$, in which the constant $c$ is taken in accordance with the chosen coverage probability.

Example 4. We determine the PDF of the collocation error in the ionospheric prediction of Example 2, cf. (10). To apply (16), we need the PMF $P[\check{x}=z]$, the mean $A_{0 \mid y}(x-z)$ and the variance $Q_{y_{0} y_{0} \mid y}$. The PMF $P[\check{x}=z]$ of integer rounding is given by the function $F(z)=\Phi\left(\frac{(1+2(x-z))}{2 \sigma_{\hat{x}}}\right)+\Phi\left(\frac{(1-2(x-z))}{2 \sigma_{\hat{x}}}\right)+1$, with $\Phi(x)=\int_{-\infty}^{x} \frac{1}{\sqrt{2 \pi}} \exp \left\{-\frac{1}{2} \omega^{2}\right\} \mathrm{d} \omega$. The function $F(z)$ is symmetric with respect to $x$ and its shape is governed by $\sigma_{\hat{x}}$. The smaller this standard deviation is, the more peaked the PMF is. For $\sigma_{\hat{x}}<0.10$, one will have $P[\check{x}=x] \approx 1$. If we denote the mean $A_{0 \mid y}(x-z)$ as $m(z)$ and the variance $Q_{y_{0} y_{0} \mid y}=$ $Q_{s_{0} s_{0}}-Q_{s_{0} s}\left(Q_{s s}+Q_{n n}\right)^{-1} Q_{s s_{0}}$ as $\sigma^{2}$, the error PDF follows as

$$
f_{\check{e}_{0}}(v)=\sum_{z \in Z} \frac{F(Z)}{\sigma \sqrt{2 \pi}} \exp \left\{-\frac{1}{2}\left(\frac{v-m(z)}{\sigma}\right)^{2}\right\}
$$

with $m(z)=-\frac{2 \lambda \sigma_{s_{0} s}}{4 \sigma_{s}^{2}+\sigma_{1}^{2}+\sigma_{2}^{2}}(x-z)$ and $\sigma^{2}=\sigma_{s_{0}}^{2}-$ $\frac{4 \sigma_{s_{0} s}^{2}}{4 \sigma_{s}^{2}+\sigma_{1}^{2}+\sigma_{2}^{2}}$. Note, since the unknown mean $x$ is an integer, that the PDF of the prediction error is independent of $x$

\section{References}

Dermanis, A. (1980): Adjustment of geodetic observations in the presence of signals. In: Proceedings of the International School of Advanced Geodesy. Bollettino di Geodesia e Scienze Affini. Vol. 38, pp. 419-445.

de Jonge P.J., C.C.J.M. Tiberius (1996): The LAMBDA method for integer ambiguity estimation: implementation aspects. Publications of the Delft Computing Centre, $L G R$ Series No. 12.

Krarup, T. (1969): A contribution to the mathematical foundation of physical geodesy. Publ. Danish Geod. Inst. 44, Copenhagen.

Moritz, H. (1973): Least-squares collocation. DGK, A 59 , Muenchen.

Moritz, H. (1980): Advanced Physical Geodesy. Herbert Wichmann Verlag Karlsruhe.

Sanso, F. (1986): Statistical methods in physical geodesy. In: Mathematical and numerical techniques in physical geodesy, H. Suenkel Ed., Lecture Notes in Earth Sciences, Springer-Verlag, Vol. 7, 49-156.

Teunissen, P.J.G. (1993): Least-squares estimation of the integer GPS ambiguities. Invited Lecture, Section IV Theory and Methodology, IAG General Meeting, Beijing, China, August 1993. Also in: LGR Series, No. 6, Delft Geodetic Computing Centre.

Teunissen, P.J.G. (1995): The least-squares ambiguity decorrelation adjustment: a method for fast GPS integer ambiguity estimation. Journal of Geodesy, 70: 65-82.

Teunissen, P.J.G. (1999): An optimality property of the integer least-squares estimator. Journal of Geodesy, 73: 587-593. 